\title{
Semistrict G-preinvexity and its application
}

\section{Zai Yun Peng*}

\section{"Correspondence:}

pengzaiyun@126.com

College of Science, Chongqing Jiao

Tong University, Chongqing,

400074, P.R. China

Department of Mathematics, Inner

Mongolia University, Hohhot,

010021, P.R. China

\begin{abstract}
In this paper, a class of semistrictly G-preinvex functions introduced by Luo and Wu (J. Comput. Appl. Math. 222:372-380, 2008) is further considered. Some properties of semistrictly G-preinvex functions are obtained, especially those containing an interesting gradient property. Then, some optimality results, which extend the corresponding results in the literature (Yang and Li in J. Math. Anal. Appl. 256:229-241, 2001; Yang and Li in J. Math. Anal. Appl. 258:287-308, 2001; Antczak in J. Glob. Optim. 43:97-109, 2009; Luo and Wu in J. Comput. Appl. Math. 222:372-380, 2008), are derived in multiobjective optimization problems.
\end{abstract}

Keywords: G-preinvex function; semistrictly G-preinvex function; optimality; multiobjective optimization

\section{Introduction}

It is well known that convexity and generalized convexity have been playing a central role in mathematical programming, economics, engineering and optimization theory. The research on characterizations and generalizations of convexity and generalized convexity is one of the most important aspects in mathematical programming and optimization theory in [1-4]. Various kinds of generalized convexity have been introduced by many authors. In 1981, Hanson [5] introduced the concept of invexity which is an extension of differentiable convex functions and proved the sufficiency of Kuhn-Tucker condition. Later, Weir and Mond [6] and Weir and Jeyakumar [7] introduced preinvex functions, and they also studied how and where preinvex functions can replace convex functions in an optimization problem. Then, Yang and Li [8] obtained some properties of a preinvex function in 2001. Yang and Li [9] also introduced the concept of semistrictly preinvex functions and investigated the relationships between semistrictly preinvex functions and preinvex functions. It is worth mentioning that many properties of invex functions and (semistrictly) preinvex functions and their applications in mathematical programming are discussed in some existing literature (see [6-11]).

On the other hand, Avriel et al. [12] introduced the definition of G-convex functions, which is another generalization of convex functions, where $G$ is a continuous real-valued increasing function. As a generalization of $G$-convex functions and invex functions, Antczak [13] introduced the concept of G-invex functions and derived some optimality conditions for constrained optimization problems under the assumption of $G$-invexity. Antczak [14] introduced a class of $G$-preinvex functions, which is a generalization of $G$ invex [13], preinvex functions [6,8] and $r$-preinvex functions [15]. Then, Luo and Wu [16] introduced the concept of semistrictly $G$-preinvex functions, which includes semistrictly 
preinvex functions [9] as a special case, and investigated the relations between semistrictly $G$-preinvex functions and $G$-preinvex functions.

However, to the best of our knowledge, it appears that there are no results on the properties and applications of semistrictly $G$-preinvex functions in literature. So, in this paper we study some properties of semistrictly $G$-preinvex functions and applications in a multiobjective optimization problem. The rest of the paper is organized as follows. In Section 2, we recall some definitions and give some examples to show that semistrictly $G$-preinvex functions are different from preinvex functions, $G$-invex functions, $G$-preinvex functions and strictly G-preinvex functions. In Section 3, we obtain some properties of semistrictly G-preinvex functions, especially those containing an interesting gradient property. Finally, optimality results for multiobjective optimization problems are obtained in Section 4. Our results extend and generalize the corresponding ones in $[8,9,13,14,16]$.

\section{Preliminaries}

Throughout this paper, let $K$ be a nonempty subset of $R^{n}$. Let $f: K \rightarrow R$ be a real-valued function and $\eta: K \times K \rightarrow R^{n}$ be a vector-valued function. And let $I_{f}(K)$ be the range of $f$, i.e., the image of $K$ under $f$, and $f^{-1}$ be the inverse of $f$.

Now we recall some definitions.

Definition 2.1 ([5]) A set $K$ is said to be invex at $y$ with respect to $\eta$ if for all $x \in K, \lambda \in[0,1]$ such that

$$
y+\lambda \eta(x, y) \in K .
$$

The set $K$ is said to be invex with respect to $\eta$ if $K$ is invex at each $y \in K$.

Definition 2.2 ([6]) Let $K \subseteq R^{n}$ be an invex set with respect to $\eta$. The function $f$ is said to be preinvex on $K$ with respect to $\eta$ iff

$$
f(y+\lambda \eta(x, y)) \leq \lambda f(x)+(1-\lambda) f(y), \quad \forall x, y \in K, \lambda \in[0,1] .
$$

Remark 2.1 Any convex function is a preinvex function with $\eta(x, y)=x-y$. But the converse is not true.

Definition 2.3 ([9]) Let $K \subseteq R^{n}$ be an invex set with respect to $\eta$. The function $f$ is said to be semistrictly preinvex on $K$ with respect to $\eta$ if, for all $x, y \in K, f(x) \neq f(y)$, we have

$$
f(y+\lambda \eta(x, y))<\lambda f(x)+(1-\lambda) f(y), \quad \lambda \in(0,1) .
$$

Remark 2.2 Any semistrictly (or strong) convex function is a semistrictly preinvex function with $\eta(x, y)=x-y$. But the converse is not true.

Definition 2.4 ([14]) Let $K \subseteq R^{n}$ be an invex set with respect to $\eta$. The function $f$ is said to be $G$-preinvex on $K$ with respect to $\eta$ if there exists a continuous real-valued increasing function $G: I_{f}(K) \rightarrow R$ such that for all $x, y \in K$ and $\lambda \in[0,1]$, we have

$$
f(y+\lambda \eta(x, y)) \leq G^{-1}(\lambda G(f(x))+(1-\lambda) G(f(y))) .
$$


$f: K \rightarrow R$ is said to be strictly $G$-preinvex on $K$, if the inequality (2.1) is strict for all $x, y \in K$, $x \neq y$ and $\lambda \in(0,1)$.

Definition 2.5 ([16]) Let $K \subseteq R^{n}$ be an invex set with respect to $\eta$. The function $f$ is said to be semistrictly $G$-preinvex on $K$ with respect to $\eta$ if there exists a continuous real-valued increasing function $G: I_{f}(K) \rightarrow R$ such that for all $x, y \in K, f(x) \neq f(y)$ and $\lambda \in(0,1)$,

$$
f(y+\lambda \eta(x, y))<G^{-1}(\lambda G(f(x))+(1-\lambda) G(f(y))) .
$$

Remark 2.3 It is clear that the semistrictly G-preinvex function is a generalization of semistrictly preinvex function.

Example 2.1 This example illustrates that a semistrictly G-preinvex function is not necessarily a (strictly) $G$-preinvex function with respect to the same $\eta$. Let

$$
\begin{aligned}
& f(x)= \begin{cases}\ln (-|x|+2) & \text { if }|x| \leq 1 ; \\
0 & \text { if }|x| \geq 1,\end{cases} \\
& \eta(x, y)= \begin{cases}x-y & \text { if } x>1,0 \leq y<1 \text {, or } x<-1,-1<y \leq 0 ; \\
x-y & \text { if } y>1,0 \leq x<1 \text {, or } y<-1,-1<x \leq 0 ; \\
1-y & \text { if } 0 \leq x<1,0<y \leq 1 ; \\
x-y & \text { if }|x| \geq 1,|y| \geq 1 ; \\
y-x & \text { if } x>1,-1<y<0 \text {, or } y>1,-1<x<0 ; \\
y-x & \text { if } x<-1,0<y<1 \text {, or } y<-1,0<x<1 ; \\
-1-\frac{1}{2} y & \text { if }|x|<1,-1 \leq y \leq 0 ; \\
x+\frac{3}{2} y & \text { if } x=1,0<y<1 \text {, or } x=-1,-1<y<0 ; \\
-y-x^{2}-x+1 & \text { if }-1<x<0,0<y \leq 1 ; \\
y-\frac{3}{2} x & \text { if } x=1,-1<y<0 \text {, or } x=-1,0 \leq y<1 .\end{cases}
\end{aligned}
$$

Then, we can verify that $f$ is a semistrictly $G$-preinvex function with respect to $\eta$, where $G(t)=e^{t}$. However, by letting $x=2, y=-\frac{3}{2}(x \neq y), \lambda=\frac{1}{2}$, we have

$$
f(y+\lambda \eta(x, y))=f\left(\frac{1}{4}\right)=\ln \frac{7}{4}>G^{-1}(\mathbf{1})=G^{-1}(\lambda G(f(x))+(1-\lambda) G(f(y))) .
$$

Thus, $f$ is not a G-preinvex function with respect to the same $\eta$, and it is also not a strictly G-preinvex function with respect to the same $\eta$.

Example 2.2 This example illustrates that a semistrictly G-preinvex function is not necessarily a $G$-invex function. Let

$$
f(x)= \begin{cases}-\ln (|x|+1) & \text { if }|x| \leq 1 \\ -\ln 2 & \text { if }|x| \geq 1\end{cases}
$$




$$
\eta(x, y)= \begin{cases}x-y & \text { if } x \geq 0, y \geq 0 \\ x-y & \text { if } x \leq 0, y \leq 0 \\ x-y & \text { if } x<-1, y>1 \\ x-y & \text { if } y<-1, x>1 \\ y-x & \text { if }-1 \leq x<0, y>0 \\ y-x & \text { if }-1 \leq y<0, x>0 \\ y-x & \text { if } 0<x \leq 1, y<0 \\ y-x & \text { if } 0<y \leq 1, x<0\end{cases}
$$

Then, by [16, Example 2], $f$ is a semistrictly $G$-preinvex function with respect to $\eta$, where $G(t)=e^{t}$. It can be easily noticed that $f$ is not differentiable at $x=0$. Thus, $f$ is not a $G$-invex function (see [13]) with respect to $\eta$.

Example 2.3 Seeing the function $f$ and $\eta$ in Example 2.1, it is obvious that $f$ is a semistrictly $G$-preinvex function with respect to $\eta$, where $G(t)=e^{t}$. However, by letting $x=4, y=-3, \lambda=\frac{1}{2}$, we have

$$
f(y+\lambda \eta(x, y))=f\left(\frac{1}{2}\right)=\ln \frac{3}{2}>\mathbf{0}=\lambda f(x)+(1-\lambda) f(y) .
$$

Thus, $f$ is not a preinvex function with respect to the same $\eta$.

Remark 2.4 From Examples 2.1-2.3, we know that semistrictly G-preinvex functions are different from $G$-preinvex functions, strictly $G$-preinvex functions, $G$-invex functions and preinvex functions with respect to the same $\eta$.

In order to discuss the properties of semistrictly G-preinvex functions, we recall the definition of Condition $\mathrm{C}$ as follows.

Condition $\mathbf{C}([8,11])$ The vector-valued function $\eta: K \times K \rightarrow R^{n}$ is said to satisfy Condition $C$ if for any $x, y \in K$ and $\lambda \in[0,1]$,

$$
\begin{aligned}
& \eta(y, y+\lambda \eta(x, y))=-\lambda \eta(x, y), \\
& \eta(x, y+\lambda \eta(x, y))=(1-\lambda) \eta(x, y) .
\end{aligned}
$$

In the sequel, we will use the following lemma.

Lemma 2.1 ([14]) $G^{-1}$ is (strictly) increasing if and only if $G$ is (strictly) increasing.

The next lemma can be easily proved by Lemma 2.1 and the definitions of a concave function and a convex function, so we omit it.

Lemma 2.2 If $G$ is increasing and concave, then $G^{-1}$ is convex.

\section{Some properties of semistrictly G-preinvex functions}

In this section, we derive some interesting properties of semistrictly $G$-preinvex functions.

Theorem 3.1 Let $K$ be a nonempty invex set with respect to $\eta$, where $\eta$ satisfies Condition $\mathrm{C}$, and let $f_{i}: K \rightarrow R(i \in I)$ be a finite or infinite collection of both semistrictly Gpreinvex and G-preinvex functions for the same $\eta$ on $K$. Define $f(x)=\sup \left\{f_{i}(x), i \in I\right\}$, for 
every $x \in K$. Assume that for every $x \in K$, there exists an $i_{0}:=i(x) \in I$ such that $f(x)=f_{i_{0}}(x)$. Then $f$ is both a semistrictly G-preinvex and G-preinvex function with respect to the same $\eta$ on $K$.

Proof By Proposition 12 in [14], we know that $f$ is $G$-preinvex on $K$. We need to show that $f$ is a semistrictly $G$-preinvex function on $K$. Assume that $f$ is not a semistrictly $G$-preinvex function. Then, there exist $x, y \in K$ with $f(x) \neq f(y)$ and $\alpha \in(0,1)$ such that

$$
f(y+\alpha \eta(x, y)) \geq G^{-1}(\alpha G(f(x))+(1-\alpha) G(f(y))) .
$$

By the G-preinvexity of $f$, we have

$$
f(y+\alpha \eta(x, y)) \leq G^{-1}(\alpha G(f(x))+(1-\alpha) G(f(y))) .
$$

It follows that

$$
f(y+\alpha \eta(x, y))=G^{-1}(\alpha G(f(x))+(1-\alpha) G(f(y))) .
$$

Let $z=y+\alpha \eta(x, y)$. By the assumptions, there exist $i(z)=i_{0}, i(x)=i_{1}, i(y)=i_{2}$, satisfying

$$
f(z)=f_{i_{0}}(z), \quad f(x)=f_{i_{1}}(x), \quad f(y)=f_{i_{2}}(y) .
$$

This fact together with (3.1) yields

$$
f_{i_{0}}(z)=G^{-1}\left(\alpha G\left(f_{i_{1}}(x)\right)+(1-\alpha) G\left(f_{i_{2}}(y)\right)\right) .
$$

(i) If $f_{i_{0}}(x) \neq f_{i_{0}}(y)$, then by the semistrict $G$-preinvexity of $f_{i_{0}}$,

$$
f_{i_{0}}(z)<G^{-1}\left(\alpha G\left(f_{i_{0}}(x)\right)+(1-\alpha) G\left(f_{i_{0}}(y)\right)\right) .
$$

From $f_{i_{0}}(x) \leq f_{i_{1}}(x), f_{i_{0}}(y) \leq f_{i_{2}}(y)$ and (3.3), we obtain

$$
f_{i_{0}}(z)<G^{-1}\left(\alpha G\left(f_{i_{1}}(x)\right)+(1-\alpha) G\left(f_{i_{2}}(y)\right)\right),
$$

which contradicts (3.2).

(ii) If $f_{i_{0}}(x)=f_{i_{0}}(y)$, then by the $G$-preinvexity of $f_{i_{0}}$,

$$
f_{i_{0}}(z) \leq G^{-1}\left(\alpha G\left(f_{i_{0}}(x)\right)+(1-\alpha) G\left(f_{i_{0}}(y)\right)\right) .
$$

Since $f(x) \neq f(y)$, at least one of the inequalities $f_{i_{0}}(x) \leq f_{i_{1}}(x)=f(x)$ and $f_{i_{0}}(y) \leq f_{i_{2}}(y)=f(y)$ has to be a strict inequality. From (3.4) and the continuity and increasing property of $G$, we obtain

$$
f(z)=f_{i_{0}}(z)<G^{-1}\left(\alpha G\left(f_{i_{1}}(x)\right)+(1-\alpha) G\left(f_{i_{2}}(y)\right)\right),
$$

which contradicts (3.2). This completes the proof. 
Remark 3.1 Theorem 3.1 generalizes Theorem 3.8 [9] from a semistrictly preinvex case to a semistrictly $G$-preinvex case.

Next, we will establish an important gradient property of semistrictly $G$-preinvex functions. Before showing the property in Theorem 3.3, we first derive a result of G-preinvex functions.

Theorem 3.2 Let $K$ be a nonempty invex set in $R^{n}$ with respect to $\eta: K \times K \rightarrow R^{n}$, and $f$ be a G-preinvex function with respect to the same $\eta$ on $K$. Assume that $\eta$ satisfies Condition $C$. For any $x, y \in K$ and $\lambda \in[0,1]$, let $g(\lambda)=G(f(x+\lambda \eta(y, x)))$. Then

$$
\frac{g(\alpha)-g(0)}{\alpha} \leq \frac{g(\beta)-g(0)}{\beta}, \quad 0<\alpha<\beta \leq 1,
$$

or equivalently,

$$
\frac{G(f(x+\alpha \eta(y, x))-G(f(x)))}{\alpha} \leq \frac{G(f(x+\beta \eta(y, x))-G(f(x)))}{\beta}, \quad 0<\alpha<\beta \leq 1 .
$$

Proof For $0<\alpha<\beta \leq 1$, let $z_{\alpha}=x+\alpha \eta(y, x), z_{\beta}=x+\beta \eta(y, x), u=1-\frac{\alpha}{\beta}$. By Condition C,

$$
\begin{aligned}
z_{\beta}+u \eta\left(x, z_{\beta}\right) & =x+\beta \eta(y, x)+u \eta(x, x+\beta \eta(y, x)) \\
& =x+(\beta-u \beta) \eta(y, x)=z_{\alpha} .
\end{aligned}
$$

We have

$$
\begin{aligned}
g(\alpha) & =G\left(f\left(z_{\alpha}\right)\right) \\
& =G\left(f\left(z_{\beta}+u \eta\left(x, z_{\beta}\right)\right)\right) \\
& \leq u G(f(x))+(1-u) G\left(f\left(z_{\beta}\right)\right) \\
& =\left(1-\frac{\alpha}{\beta}\right) g(0)+\frac{\alpha}{\beta} g(\beta) .
\end{aligned}
$$

Therefore, we obtain

$$
\frac{g(\alpha)-g(0)}{\alpha} \leq \frac{g(\beta)-g(0)}{\beta}, \quad 0<\alpha<\beta \leq 1 .
$$

This completes the proof.

From Definitions 2.4 and 2.5, we can obtain the following lemma.

Lemma 3.1 Let $K$ be a nonempty invex set with respect to $\eta$, where $\eta$ satisfies Condition C. Let $f: K \rightarrow R$ be continuous and semistrictly G-preinvex with respect to $\eta$ on $K$, and satisfy $f(y+\eta(x, y)) \leq f(x)(\forall x \in K)$. Then $f$ is a G-preinvex function on $K$.

Theorem 3.3 Let $K$ be a nonempty invex set with respect to $\eta$, where $\eta$ satisfies Condition C. Assume that $f: K \rightarrow R$ is differentiable and semistrictly G-preinvex with respect to $\eta$ on $K$, and satisfies $f(y+\eta(x, y)) \leq f(x)(\forall x \in K)$, where $G$ is a differentiable function. Then for any $x, y \in K$ with $f(x) \neq f(y)$, we have 
(i) $G(f(y))>G(f(x))+G^{\prime}(f(x)) \eta(y, x)^{T} \nabla f(x)$,

(ii) $G^{\prime}(f(x)) \eta(y, x)^{T} \nabla f(x)+G^{\prime}(f(y)) \eta(x, y)^{T} \nabla f(y)<0$.

Proof (i) Suppose that $f$ is a semistrictly G-preinvex function on $K$. By Definition 2.4, for any $x, y \in K$ with $f(x) \neq f(y)$, we have

$$
f(x+\lambda \eta(y, x))<G^{-1}(\lambda G(f(y))+(1-\lambda) G(f(x))), \quad \forall \lambda \in(0,1),
$$

which implies

$$
G(f(x+\lambda \eta(y, x)))<\lambda G(f(y))+(1-\lambda) G(f(x)) .
$$

It follows that

$$
\frac{G(f(x+\lambda \eta(y, x))-G(f(x)))}{\lambda}<G(f(y))-G(f(x)) .
$$

From Lemma 3.1 and Theorem 3.2, we get

$$
G^{\prime}(f(x)) \eta(y, x)^{T} \nabla f(x)=\inf _{\lambda \geq 0} \frac{G(f(x+\lambda \eta(y, x))-G(f(x)))}{\lambda}<G(f(y))-G(f(x)),
$$

that is

$$
G(f(y))>G(f(x))+G^{\prime}(f(x)) \eta(y, x)^{T} \nabla f(x) .
$$

(ii) Since $f$ is a semistrictly $G$-preinvex function on $K$, for $x, y \in K$ with $f(x) \neq f(y)$, it follows form the above results that

$$
\begin{aligned}
& G(f(y))>G(f(x))+G^{\prime}(f(x)) \eta(y, x)^{T} \nabla f(x) . \\
& G(f(x))>G(f(y))+G^{\prime}(f(y)) \eta(x, y)^{T} \nabla f(y) .
\end{aligned}
$$

From (3.5) and (3.6), we can obtain

$$
G^{\prime}(f(x)) \eta(y, x)^{T} \nabla f(x)+G^{\prime}(f(y)) \eta(x, y)^{T} \nabla f(y)<0 .
$$

This completes the proof.

Remark 3.2 As a matter of fact, the assumption of continuity for $f$ can be extended to lower semicontinuity in Lemma 3.1 .

\section{Semistrict G-preinvexity and optimality}

In the section, we consider a class of multiobjective optimization problems and obtain an important optimality result under semistrict $G$-preinvexity.

From now on, we suppose that $f=\left(f_{1}, \ldots, f_{n}\right): X \rightarrow R^{n}$ is a vector-valued mapping, where $X$ is an invex subset of $R^{n}$ endowed with the Euclidean norm $\|\cdot\|$. 
We consider the following multiobjective optimization problem:

$$
\begin{gathered}
\left(\text { MOP): } \operatorname{minimize} f(x)=\left(f_{1}(x), f_{2}(x), \ldots, f_{p}(x)\right)\right. \\
\text { subject to } x \in X, \\
\text { where } f_{i}: X \rightarrow R, i=1,2, \ldots, n .
\end{gathered}
$$

In the sequel, we use the following notations. For $x, y \in X$

(i) $f(x)<f(y) \Longleftrightarrow f_{i}(x)<f_{i}(y)$ for every $i=1,2, \ldots, n$;

(ii) $f(x) \nless f(y)$ is the negation of $f(x)<f(y)$.

Definition 4.1 Let $m \geq 1$ be an integer. A point $\bar{x} \in X$ is said to be a strictly local minimizer of order $m$ for (MOP) if there exist an $\varepsilon>0$ and a vector $c \in \operatorname{int} R_{+}^{n}$ such that

$$
f(x) \nless f(\bar{x})+c\|\eta(x, \bar{x})\|^{m}, \quad \forall x \in N(\bar{x}, \varepsilon) \cap X,
$$

where $N(\bar{x}, \varepsilon)$ is an $\varepsilon$ neighbor of $\bar{x}$.

Now, we give the notion of a strict minimizer in the global sense if the neighbor $N(\bar{x}, \varepsilon)$ is replaced by the whole space $R^{n}$.

Definition 4.2 Let $m \geq 1$ be an integer. A point $\bar{x} \in X$ is said to be a strictly global minimizer of order $m$ for (MOP) if there exists a vector $c \in \operatorname{int} R_{+}^{n}$ such that

$$
f(x) \nless f(\bar{x})+c\|\eta(x, \bar{x})\|^{m}, \quad \forall x \in X .
$$

Remark 4.1 If $\eta(x, y)=x-y$, then Definitions 4.1-4.2 reduce to Definitions 4.2-4.3 introduced by Bhatia [17], respectively.

Remark 4.2 From Definitions 4.1 and 4.2, we know that the concepts of a strictly local minimizer of order $m$ and a strictly global minimizer of order $m$ for (MOP) are stronger than the concepts of a strictly local minimizer and a strictly global minimizer for (MOP), respectively.

It is clear that any strictly global minimizer of order $m$ is a strictly global minimizer. But the converse may not be true. We can see the case in the following example.

Example 4.1 Let $f: R \rightarrow R^{3}$ be defined as

$$
f(x)=\left(f_{1}(x), f_{2}(x), f_{3}(x)\right)= \begin{cases}\left(-x, x^{\frac{1}{x}},-\frac{1}{4} x\right) & \text { if } x>0 \\ (0,0,0) & \text { if } x=0\end{cases}
$$

Consider the multiobjective optimization problem,

$$
\operatorname{minimize} f(x)
$$


$\bar{x}=0$ is a strictly global minimizer but is not a strictly global minimizer of order $m$, because for any $m>0, c=\left(c_{1}, c_{2}, c_{3}\right) \in \operatorname{int} R_{+}^{3}$ and $x>0$ sufficiently small, we have $f(x)<c x^{m}$, where $\eta(x, y)=x-y$ or $\eta(x, y)=x+y$.

Theorem 4.1 Let $X$ be an invex set with respect to $\eta$. Suppose the following conditions are satisfied:

(i) Let $G$ be increasing and concave on $I_{f}(X)$;

(ii) $\eta: X \times X \rightarrow R^{n}$ satisfies Condition $\mathrm{C}$;

(iii) $\bar{x} \in X$ is a strictly local minimizer of order $m$ for $(M O P)$.

If $f_{i}: X \rightarrow R, i=1,2, \ldots, n$, are semistrictly $G$-preinvex on $X$ with respect to $\eta$, then $\bar{x}$ is a strictly global minimizer of order $m$ for $(M O P)$.

Proof Let $\bar{x} \in X$ be a strictly local minimizer of order $m$ for (MOP). Then there exist an $\varepsilon$ neighborhood $N(\bar{x}, \varepsilon)$ of $\bar{x}$ and a vector $c \in \operatorname{int} R_{+}^{n}$ such that

$$
f(x) \nless f(\bar{x})+c\|\eta(x, \bar{x})\|^{m}, \quad \forall x \in N(\bar{x}, \varepsilon) \cap X .
$$

Hence, there exists no $x \in N(\bar{x}, \varepsilon) \cap X$ such that

$$
f_{i}(x)<f_{i}(\bar{x})+c_{i}\|\eta(x, \bar{x})\|^{m}, \quad i=1,2, \ldots, n
$$

where $c=\left(c_{1}, c_{2}, \ldots, c_{n}\right)$.

Suppose by contradiction that $\bar{x}$ is not a strictly global minimizer of order $m$ for (VP), then there exists $x^{*} \in X$ with $f\left(x^{*}\right) \neq f(\bar{x})$ such that

$$
f_{i}\left(x^{*}\right)<f_{i}(\bar{x})+c_{i}^{*}\left\|\eta\left(x^{*}, \bar{x}\right)\right\|^{m}, \quad i=1,2, \ldots, n
$$

for any $c^{*}=\left(c_{1}^{\prime \prime}, c_{2}^{*}, \ldots, c_{n}^{* \prime}\right) \in \operatorname{int} R_{+}^{n}$. Since $X$ is an invex set with respect to $\eta$,

$$
\bar{x}+\lambda \eta\left(x^{\prime \prime}, \bar{x}\right) \in X, \quad \forall \lambda \in(0,1) .
$$

Because $f_{i}: X \rightarrow R, i=1,2, \ldots, n$, are semistrictly $G$-preinvex on $X$ with respect to $\eta, G$ is increasing on $I_{f}$ and $G^{-1}$ is convex, it follows that for any $\lambda \in(0,1)$

$$
\begin{aligned}
f_{i}\left(\bar{x}+\lambda \eta\left(x^{* \prime}, \bar{x}\right)\right) & <G^{-1}\left(\lambda G\left(f_{i}\left(x^{\prime \prime}\right)\right)+(1-\lambda) G\left(f_{i}(\bar{x})\right)\right) \\
& \leq \lambda f_{i}\left(x^{*}\right)+(1-\lambda) f_{i}(\bar{x}),
\end{aligned}
$$

where Lemma 2.2 is used in the second inequality.

According to (4.2), (4.3) and Condition C, we have

$$
\begin{aligned}
f_{i}\left(\bar{x}+\lambda \eta\left(x^{*}, \bar{x}\right)\right)-f_{i}(\bar{x}) & \leq \lambda\left(f_{i}\left(x^{*}\right)-f_{i}(\bar{x})\right) \\
& <\lambda c_{i}^{*}\left\|\eta\left(x^{*}, \bar{x}\right)\right\|^{m} \\
& =\lambda^{1-m} c_{i}^{*}\left\|\eta\left(\bar{x}+\lambda \eta\left(x^{*}, \bar{x}\right), \bar{x}\right)\right\|^{m} \\
& =d_{i}\left\|\eta\left(\bar{x}+\lambda \eta\left(x^{*}, \bar{x}\right), \bar{x}\right)\right\|^{m},
\end{aligned}
$$


where $d_{i}=\lambda^{1-m} c_{i}^{*}$. For a sufficiently small $\lambda>0$, we obtain

$$
\bar{x}+\lambda \eta\left(x^{*}, \bar{x}\right) \in X \cap N(\bar{x}, \varepsilon) .
$$

Let $d=\left(d_{1}, \ldots, d_{n}\right)$. Since $c^{*}=\left(c_{1}^{*}, c_{2}^{*}, \ldots, c_{n}^{*}\right) \in \operatorname{int} R_{+}^{n}$ is arbitrary, $d=\left(d_{1}, \ldots, d_{n}\right) \in \operatorname{int} R_{+}^{n}$ is also arbitrary. Therefore,

$$
f\left(\bar{x}+\lambda \eta\left(x^{*}, \bar{x}\right)\right)-f(\bar{x})<d\left\|\eta\left(\bar{x}+\lambda \eta\left(x^{*}, \bar{x}\right), \bar{x}\right)\right\|^{m}, \quad \forall d \in \operatorname{int} R_{+}^{n},
$$

or

$$
f_{i}\left(\bar{x}+\lambda \eta\left(x^{*}, \bar{x}\right)\right)<f_{i}(\bar{x})+d_{i}\left\|\eta\left(\bar{x}+\lambda \eta\left(x^{*}, \bar{x}\right), \bar{x}\right)\right\|^{m}, \quad i=1,2, \ldots, n
$$

which implies that $\bar{x}$ is not a strictly local minimizer of order $m$. It is a contradiction. Hence, $\bar{x}$ is a strict minimizer of order $m$ for (MOP).

Now, we give an example of an optimization problem to illustrate Theorem 4.1.

Example 4.2 Let $f: R \rightarrow R^{3}$ be defined as

$$
f(x)= \begin{cases}(\ln (-2|x|+3), \ln (-2|x|+3), \ln (-2|x|+3)) & \text { if }|x| \leq 1 \\ (0,0,0) & \text { if }|x| \geq 1\end{cases}
$$

where

$$
\begin{aligned}
& f_{i}(x)= \begin{cases}\ln (-2|x|+3) & \text { if }|x| \leq 1, \quad(i=1,2,3) \\
0 & \text { if }|x| \geq 1\end{cases} \\
& \eta(x, y)= \begin{cases}x-y & \text { if } x>1,0 \leq y<1 \text {, or } x<-1,-1<y \leq 0 \\
x-y & \text { if } y>1,0 \leq x<1 \text {, or } y<-1,-1<x \leq 0 \\
1-y & \text { if } 0 \leq x<1,0<y \leq 1 \\
x-y & \text { if }|x| \geq 1,|y| \geq 1 ; \\
y-x & \text { if } x>1,-1<y<0 \text {, or } y>1,-1<x<0 \\
-1-\frac{1}{2} y & \text { if } x<-1,0<y<1 \text {, or } y<-1,0<x<1 \\
x+\frac{3}{2} y & \text { if }|x|<1,-1 \leq y \leq 0 ; \\
-y-x^{2}-x+1 & \text { if } x=1,0<y<1 \text {, or } x=-1,-1<y<0 \\
y-2 x & \text { if }-1<x<0,0<y \leq 1\end{cases}
\end{aligned}
$$

From Definition 2.5 , we can verify that $f_{i}(i=1,2,3)$ are semistrictly $G$-preinvex functions with respect to $\eta$, where $G(t)=e^{t} \cdot \bar{x}=1$ is a strictly local minimizer of order $m$ for (MOP). From Theorem 4.1, we can get $\bar{x}=1$ is also a strictly global minimizer of order $m$ for (MOP), and $f(\bar{x})=(0,0,0)$ is a global minimal value of (MOP). 


\section{Acknowledgements}

The author is very grateful to the three anonymous referees for valuable comments and suggestions which helped to improve the paper. This work was supported by the Natural Science Foundation of China (No. 11271389, 11201509, 71271226), the Natural Science Foundation Project of Chongqing (No. CSTC, 2011AC6104.2012jjA00016) and the Education Committee Project Research Foundation of Chongqing (No. KJ100711).

Received: 4 April 2012 Accepted: 6 August 2012 Published: 7 September 2012

\section{References}

1. Mangasarin, OL: Nonlinear Programming. Mcgraw-Hill, New York (1969)

2. Bazaraa, MS, Sherali, HD, Shetty, CM: Nonlinear Programming Theory and Algorithms. Wiley, New York (1979)

3. Schaible, S, Ziemba, WT: Generalized Concavity in Optimization and Economics. Academic Press, London (1981)

4. Ward, DE: Characterizations of strict local minima and necessary conditions for weak sharp minima. J. Optim. Theory Appl. 80, 551-571 (1994)

5. Hanson, MA: On sufficiency of Kuhn-Tucker conditions. J. Math. Anal. Appl. 80, 545-550 (1981)

6. Weir, T, Mond, B: Pre-invex functions in multiple objective optimization. J. Optim. Theory Appl. 136, 29-38 (1988)

7. Weir, T, Jeyakumar, V: A class of nonconvex functions and mathematical programming. Bull. Aust. Math. Soc. 38 , 177-189 (1988)

8. Yang, XM, Li, D: On properties of preinvex functions. J. Math. Anal. Appl. 256, 229-241 (2001)

9. Yang, XM, Li, D: Semistrictly preinvex functions. J. Math. Anal. Appl. 258, 287-308 (2001)

10. Yang, XM, Yang, XQ, Teo, KL: Characterizations and applications of prequasi-invex functions. J. Optim. Theory Appl. $110,645-668(2001)$

11. Peng, JW, Yang, XM: Two properties of strictly preinvex functions. Oper. Res. Trans. 9, 37-42 (2005)

12. Avriel, M, Diewert, WE, Schaible, S, Zang, I: Generalized Concavity. Plenum, New York (1975)

13. Antczak, T: On G-invex multiobjective programming. I. Optimality. J. Glob. Optim. 43, 97-109 (2009)

14. Antczak, T: G-preinvex functions in mathematical programming. J. Comput. Appl. Math. 217, $212-226$ (2008)

15. Antczak, T: $r$-preinvexity and $r$-invexity in mathematical programming. Comput. Math. Appl. 50, 551-566 (2005)

16. Luo, HZ, Wu, HX: On the relationships between G-preinvex functions and semistrictly G-preinvex functions. J. Comput. Appl. Math. 222, 372-380 (2008)

17. Bhatia, G: Optimality and mixed saddle point criteria in multiobjective optimization. J. Math. Anal. Appl. 342, 135-145 (2008)

doi:10.1186/1029-242X-2012-198

Cite this article as: Peng: Semistrict G-preinvexity and its application. Journal of Inequalities and Applications 2012

2012:198.

\section{Submit your manuscript to a SpringerOpen ${ }^{\ominus}$ journal and benefit from:}

- Convenient online submission

- Rigorous peer review

- Immediate publication on acceptance

Open access: articles freely available online

- High visibility within the field

- Retaining the copyright to your article 